\title{
Seismic constraints on a model of partial melts under ridge axes
}

\author{
Toshiro Tanimoto \\ Institute of Crustal Studies and Department of Geological Sciences, University of California, Santa Barbara
}

\author{
David J. Stevenson \\ Division of Geological and Planetary Sciences, California Institute of Technology, Pasadena
}

\begin{abstract}
In a recent global scale seismic study, the correlation between $S$ wave velocity under ridge axes and spreading rate was pointed out. The correlation is strong for depths to about $70 \mathrm{~km}$, but it diminishes below this depth. We present the correlation plots at four depths, $38,66,90$, and $110 \mathrm{~km}$, for which correlation is strong at 38 and $66 \mathrm{~km}$ but is weak at $90 \mathrm{~km}$ and is almost nonexistent at $110 \mathrm{~km}$. We present a model to explain this behavior, which includes a thermal conduction model for the development of lithosphere and a simple melt percolation. Thermal effects on $S$ wave velocity are assumed to be accounted for entirely by the plate cooling (thermal conduction) model. We point out that the thermal model under this assumption predicts asymptotically no correlation between $S$ wave velocity and spreading rate, specifically for spreading rate larger than about $3 \mathrm{~cm} \mathrm{yr}^{-1}$. This contradicts the correlation observed in the data at shallow depths. The existence of partial melt is thus required to explain the observed behavior at 38 and $66 \mathrm{~km}$ depths. We start from four basic equations that govern the distribution of partial melt and derive the relation between the amount of partial melt and the spreading rate. We adopt a simple power law relation between permeability $(k)$ and porosity $(f)$ by $k(f)=k_{0} f^{n}$, where $k_{0}$ and $n$ are constants and assume that pores are filled with melt. We then set up an integral relation between $S$ wave velocity and spreading rate. The final formula indicates that the gradient in the correlation plots is the inverse of the power $(1 / n)$ in the permeability-porosity relation, thus enabling us to constrain $n$ as well as $k_{0}$ from seismic data. The data also have some sensitivity to the depth to solidus. We show that (1) the depth to solidus is probably within the range $60-100 \mathrm{~km}$ and (2) if the power $n$ is $n=2-3$, then $k_{0}=10^{-8}-10^{-10} \mathrm{~m}^{2}$. These parameters predict that porosity and fluid velocity are $1-2 \%$ and about $0.5 \mathrm{~m} \mathrm{yr}^{-1}$, respectively. The depth to solidus is consistent with previous estimates by petrological data but is perhaps the first and direct seismological evidence of partial melt from surface wave data. Analytical forms for the dependence on depth and spreading rate of porosity, fluid velocity within permeable rocks, and ascent times of magma are also obtained.
\end{abstract}

\section{Introduction}

In order to understand the genesis of oceanic crust and mid-ocean ridge basalt magma (MORB), it is essential to know the pattern of flow as well as distribution of partial melts under the ridge axes. However, there are currently very few observational constraints available, and it seems important to examine any available data that can address these questions.

Global seismic structure studies of the past decade have revealed slow-velocity anomalies along ridge axes [e.g., Woodhouse and Dziewonski, 1984; Nataf et al., 1986; Tanimoto, 1988, 1990]. Depth resolution, especially within the upper $200 \mathrm{~km}$, was greatly improved by a recent study [Zhang and Tanimoto, 1992, 1993]. In the latter results, lateral extents of slow-velocity anomalies correlate with spreading rates and $S$ wave velocity becomes progressively slower with shallower depth, which suggests increasing amount of melt under faster spreading ridges.

Copyright 1994 by the American Geophysical Union.

Paper number 93JB03402.

0148-0227/94/93JB-03402\$05.00
Other seismological attempts include, for example, Sheehan and Solomon [1991] and Woodward and Masters [1991]. It is generally believed, however, that seismic data alone cannot constrain temperature and partial melt distribution simultaneously. Because of this, some researchers have combined both seismic properties and electrical properties in order to constrain the thermal state as well as the partial melt quantity in the upper mantle [e.g., Shankland et al., 1981]. Others have applied experimental laboratory data to interpret the seismic results, using the idea of extrapolation of homologous temperature [Sato et al., 1989]. Recently, Forsyth [1922] published a comprehensive review on this subject, specifically on observational constraints on mantle flow and melt distribution under ridge axes.

In this paper, we take a fresh approach and focus on a particular aspect of global seismic velocity variations: $S$ wave velocity variations under ridge axes as a function of spreading rates. We point out that the trend in the $S$ wave velocity variation under ridge axes provides critical information in distinguishing partial melt effects from thermal effects. The information is in the asymptotic behavior of seismic velocity as spreading rate increases. In the thermal 
model, the seismic velocity at a given depth beneath the ridge axes should approach a constant value with increasing spreading rate. In the partial melting model, if permeability $k$ is given as a function of porosity $f$ by $k(f)=k_{0} f^{n}$, where $k_{0}$ and $n$ are constants, the asymptotic variation of seismic velocity with spreading rate should be related to the exponent $n$ (actually $1 / n$ ). Thus it becomes possible to distinguish partial melt effects as long as $n$ is not large. Furthermore, we can determine $k_{0}$ and the depth to the solidus. In the following, we first discuss the theoretical background and then show the results of the data analysis.

\section{Seismic Parameter}

The basic data we examine is the $S$ wave velocity perturbations from the global average at each depth. Let us first consider the relation between $S$ wave velocity perturbation and temperature $(T)$ and porosity or melt fraction $(f)$. For a list of symbols refer to the notation list.

Let $\beta$ be $S$ wave velocity and write

$$
\beta=\beta(T, f) .
$$

We drop compositional variations from our consideration. We also ignore phase transitions that are expected to occur at two depths within the depth range of consideration [Wood and Yuen, 1983]. However, considering the strong effects of partial melts on $S$ wave velocity, compositional variations and phase transitions are not likely to be as important. Effects of pressure are avoided almost entirely because we form relative ratios of quantities at the same depth.

Let us write the global average of $S$ wave velocity at each depth by $\beta_{g}$. Assuming that first-order approximation is sufficiently accurate, we can write

$\beta(T, f)=\beta_{g}\left(T_{0}, 0\right)+\left(T-T_{0}\right)\left(\frac{\partial \beta}{\partial T}\right)_{f}+f\left(\frac{\partial \beta}{\partial f}\right)_{T}$,

where $T_{0}$ is the global average temperature at each depth and is assumed to be below the solidus. There is a subtle point in taking $T$ and $f$ as independent parameters, because $f$ should be zero if $T$ is below the solidus. However, in a partially molten medium, they have two almost independent effects on the seismic velocity: Variation in $T$ is used to characterize the change in elastic properties of the solid matrix, and variation in $f$ characterizes the change in the mean elastic properties of the entire system (melt and solid) due to the presence of interpenetrating tubules of melt [cf. Mavko, 1980].

We will focus on the parameter $\varepsilon$ defined by

$$
\varepsilon=-\frac{\beta(T, f)-\beta_{g}\left(T_{0}, 0\right)}{\beta_{g}\left(T_{0}, 0\right)} .
$$

Then we can write

$$
\varepsilon=-\left(T-T_{0}\right)\left(\frac{\partial \ln \beta}{\partial T}\right)_{f}-f\left(\frac{\partial \ln \beta}{\partial f}\right)_{T} \equiv \varepsilon_{T}+\varepsilon_{f},
$$

where we introduced $\varepsilon_{T}$ and $\varepsilon_{f}$. The first term $\varepsilon_{T}$ represents the effects due to temperature deviation from the global average, and the second term $\varepsilon_{f}$ represents the effects due to partial melts. The notation ln refers to natural logarithm. Note that we use the negative sign on the right-hand side of
(3) which makes $\varepsilon$ mostly positive under ridge axes. The parameter $f$ is the perturbation from the reference state because the global average is assumed to be below the solidus.

In seismic structure studies, resolution is not perfect because of the finite amount of data (incomplete coverage over the area of study) and data errors. Equation (4) cannot be applied directly to interpret data, since the effect is not taken into account. We modify (4) so that it explicitly includes the effect of finite resolution. Let us write the resolution kernels from seismic study by $K(x, y, z)$. Then $\varepsilon_{T}$ and $\varepsilon_{f}$ should be written as

$$
\varepsilon_{T}=-\int d v\left(T-T_{0}\right)\left(\frac{\partial \ln \beta}{\partial T}\right)_{f} K(x, y, z)
$$

and

$$
\varepsilon_{f}=-\int d v f\left(\frac{\partial \ln \beta}{\partial f}\right)_{T} K(x, y, z),
$$

rather than the above definitions. Hereafter, we use (5) and (6) as definitions for $\varepsilon_{T}$ and $\varepsilon_{f}$. The integrations are carried out with respect to the volume of the Earth. In this study, $K$ has a lateral diameter of $800-1000 \mathrm{~km}$ and vertical extent of $70-100 \mathrm{~km}$ with some spatial dependence. Inclusion of the resolution kernel in the analysis reduces many complex issues in modeling that result from spatial variation of resolution; for example, measured $S$ wave velocity at slow spreading ridges is biased to higher velocity due to thicker (higher velocity) lithosphere in its neighborhood. Measured velocity, say, at $38 \mathrm{~km}$, corresponds to sharper resolution than velocity at $110 \mathrm{~km}$. All these effects are included in the modeling when the above integrations are carried out with seismically determined resolution kernels.

We will examine $\varepsilon$ only under ridge axes. The key point is to understand how this varies as a function of spreading rates. In the following, we will derive expressions for $\varepsilon_{T}$ and $\varepsilon_{f}$ for further data analyses.

\section{$\varepsilon_{T}$ : Thermal Effects}

The conductive cooling model for the development of lithosphere has been quite successful in explaining ocean floor topography, heat flow data, and surface wave dispersion data [e.g., Parsons and Sclator, 1977]. We will adopt this model to evaluate thermal effects.

It is clear now, however, that there are variations along the ridge axes, which require three-dimensional understanding of ridge structure. The data to support this come from geochemistry [e.g., Klein and Langmuir, 1987] and from topography [e.g., Hayes, 1988]. Our data show, however, that the spreading rate is the critical parameter in determining trends in shallow $S$ wave velocity structure. Threedimensional effect is clearly a higher-order effect and shows up in our current data as a scatter of points.

Temperature structure in the conductive cooling model is given by

$$
T=T_{s}+\left(T_{m}-T_{s}\right) \operatorname{erf}\left(\frac{z}{2 \sqrt{\kappa x / u}}\right)
$$

where $T_{s}$ and $T_{m}$ are surface and mantle temperature (asthenosphere), erf is the error function, $\kappa$ is the thermal 
diffusivity of lithosphere, $x$ is the distance from the ridge axis and $u$ is the half-spreading rate. Even though we examine $\varepsilon$ under the ridge axis $(x=0)$, this formula is necessary because finite resolution of data requires us to evaluate effects from regions other than $x=0$.

For our purposes, we need an expression for $T-T_{0}$, that is given by

$T-T_{0}=\left(T_{m}-T_{0}\right)-\left(T_{m}-T_{s}\right) \operatorname{erfc}\left(\frac{z}{2 \sqrt{\kappa x / u}}\right)$.

$T_{m}-T_{s}$ is generally assumed to be $1200^{\circ}-1300^{\circ} \mathrm{C}$, but we must also get $T_{0}$ in order to evaluate this formula. Assumption for geotherm could be used for this, but uncertainties may be large. In the data analysis, we will thus form relative data for $\varepsilon$, that is, the difference of $\varepsilon$ between the data for spreading rate $u$ and that at the reference $u_{\text {ref }}$, so that we can avoid the first term on the right-hand side in order to evaluate thermal effects.

When the argument of the complementary error function is larger than about unity, this function takes the form of a Gaussian tail and is accordingly very small. The argument can be written in the form

$$
\frac{z}{2 \sqrt{\kappa x / u}} \approx 3\left(\frac{z}{60 \mathrm{~km}}\right)\left(\frac{100 \mathrm{~km}}{x}\right)^{1 / 2}\left(\frac{u}{3 \mathrm{~cm} \mathrm{yr}^{-1}}\right)^{1 / 2}
$$

assuming $\kappa=0.01 \mathrm{~cm}^{2} \mathrm{~s}^{-1}$. On the right-hand side, $z$ and $x$, have the dimension of kilometers, while $u$ has the dimension of centimeters per year. This shows that for fast spreading ridges, for example, the effects of thermal diffusion have not significantly penetrated to $60 \mathrm{~km}$ depth even at $200 \mathrm{~km}$ off-axis, because the argument is larger than 1. In such a case, $T-T_{0}=T_{m}-T_{0}$ to a good approximation, and $\varepsilon_{T}$ becomes independent of $u$. Naturally, if $\varepsilon$ were dominated by thermal effects, then we should expect to see a constant asymptote for $\varepsilon$ as $u$ increases. As we show in the data analysis below, this is clearly violated by the data, indicating the need for partial melting.

\section{$\varepsilon_{f}$ : Partial Melt Effects}

In order to construct a model for partial melting, we use four basic equations: (1) an equation for melt generation, (2) mass balance, (3) continuity for melt, and (4) Darcy's law.

The melt generation depends on the rate at which an adiabatic parcel of rising mantle crosses phase equilibria curves. Strictly speaking, this is a complex issue since the system is neither one where we can assign a single melting "point" (the solidus) and evaluate melt production purely by latent heat considerations [cf. Turcotte and Ahern, 1978], nor can we compute the melt fraction as though the melt did not migrate (the usual laboratory technique for estimating melting as a function of supersolidus temperature). In the absence of a more complete understanding of the phase equilibria, especially for small melt fractions, we will adopt the following very simple linear parametrization

$$
m(z)=m_{0}\left(1-z / z_{0}\right)
$$

[Scott and Stevenson, 1989], where $m$ is the degree of melting, $m_{0}$ is a constant, and $z_{0}$ is the depth to the solidus. By "degree of melting," we mean the volume fraction of initially fertile peridotite that has melted upon traversing a path. We further assume $m_{0} \approx 0.25$, which implies that, were a fertile peridotitic parcel transported from the mean mantle adiabat right to the surface without heat loss, then one quarter of it would have melted. Notice that $m(z) \equiv$ $f(z)$ if there is no melt migration (i.e., zero permeability). The linear choice for $m(z)$ is consistent with the prediction of a univariant phase diagram [Turcotte and Ahern, 1978] and also crudely consistent with the laboratory melting behavior of peridotite [Sato et al., 1989]. Use of (10) in our analysis shows that our approach is a phenomenological one rather than the one from first principles. The approach from first principles may be more desirable, but in view of the quality of data and of modeling with a simple twodimensional model (three-dimensionality only comes from differences in spreading rates in our modeling), this approach may be justified.

The second equation, the equation for mass balance, is written as

$$
\rho_{s} v_{0}=\rho_{l} v_{l} f+\rho_{s} v_{s}(1-f),
$$

where $v_{0}$ is the mantle flow velocity below the solidus, $v_{s}$ and $v_{l}$ are the mean vertical velocities of solid and liquid, respectively, and $\rho_{s}$ and $\rho_{l}$ are densities of solid and liquid, respectively. This equation shows the balance of mass between the two media above and below the depth of solidus. It assumes one-dimensional flow. We also assume that $v_{0}$ is a simple, specified flow, which means we ignore the additional dynamical flows studied by Scott and Stevenson [1989].

The third equation, the equation for continuity of melt flux alone, is given by

$$
\frac{d}{d z}\left(f \rho_{l} v_{l}\right)=\frac{d}{d z}\left(m \rho_{s} v_{s}\right)
$$

or

$$
f v_{l}=\frac{\rho_{s}}{\rho_{l}} m v_{s}
$$

where the rest frame is the mean rest frame of the mantle and the constant of integration can be shown to be zero from the condition at depth of solidus. Partial melt is created at all depths and migrates upward immediately because of its low density. This equation assures continuity of melt under such conditions.

The fourth type of equation is Darcy's law, which takes the form

$$
f\left(v_{l}-v_{s}\right)=k(f) g \Delta \rho / \eta_{l}
$$

where $\Delta \rho \equiv \rho_{s}-\rho_{l}, k(f)$ is the permeability and $\eta_{l}$ is the fluid (magma) viscosity. We take a power law form for the permeability $k(f)=k_{0} f^{n}$, where $k_{0}$ and $n$ are constants. This ignores the possibility of any "trapped melt" (i.e., $k \equiv$ 0 until $f>f_{L}$, some critical melt fraction) [Toramaru and Fujii, 1986]. Existence of trapped melt should be rare, since it is detected by seismology as very low velocity anomalies, but evidence of such anomalies has not been often reported. Our basic assumption is that as soon as melt pockets are created, they connect immediately and upward flow of melt begins.

Solving (10), (11), (13), and (14), we get 


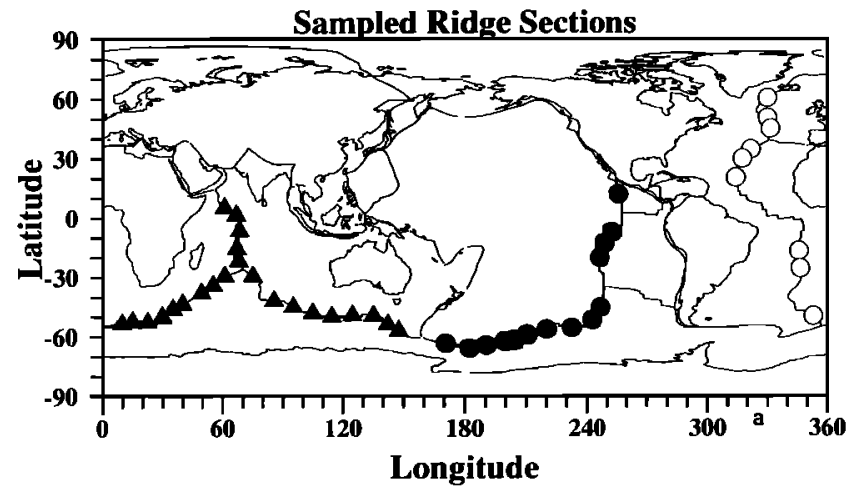

Figure 1a. Sampled ridge locations. Solid circles are the locations in the Pacific Ocean, triangles are locations in the Indian Ocean, and open circles are locations in the Atlantic Ocean. The same convention is kept in Figure lb. There are 45 locations in this figure. The data with spreading rate less than $2 \mathrm{~cm} \mathrm{yr}^{-1}$ are not relevant to our analysis, however. Latitudes and longitudes at the center of symbols are given in Table 2.

$$
\begin{gathered}
v_{s}=v_{0} /(1+m-f) \\
v_{l}=\rho_{s} m v_{0} / \rho_{l} f(1+m-f),
\end{gathered}
$$

and $f\left(v_{l}-v_{s}\right) \approx m v_{0}$, provided we ignore $f$ relative to $m$, set $\rho_{s} / \rho_{l} \approx 1$ and assume both $f$ and $m$ are small compared to unity. These are adequately accurate approximations given the level of accuracy that we need. Finally, we can invert Darcy's law for $f(z)$ :

$$
f(z) \approx\left\{\frac{m_{0} \eta_{0} v_{0}}{k_{0} \Delta \rho g}\left(1-\frac{z}{z_{0}}\right)\right\}^{1 / n} .
$$

In fact, $v_{0}$ is a function of $x$ as well as $z$. We adopt a very simple model in which the standard corner flow [e.g., Spiegelman and McKenzie, 1987] is replaced by a sharp corner flow; that is,

$$
\begin{array}{ll}
v_{0}(x, z)=0, & z<|x| \\
v_{0}(x, z)=v_{0}, & z \geq|x|
\end{array}
$$

Then for $\varepsilon_{f}$, we obtain

$$
\begin{array}{r}
\varepsilon_{f}=\left\{\frac{m_{0} \eta_{l} v_{0}}{k_{0} \Delta \rho g}\right\}^{1 / n} \int d v K\left(\frac{\partial \ln \beta}{\partial f}\right)_{T}\left(1-\frac{z}{z_{0}}\right)^{1 / n} \\
\cdot \Theta(z-|x|) \Theta\left(z_{0}-z\right),
\end{array}
$$

where $\Theta$ is the Heaviside step function. The two-step functions in this formula indicates that the zone of partial melt in this model is confined to a triangular region bounded by a surface $z=z_{0}$, and two other surfaces which intercept at the mid-ocean ridge ( $x=0, z=0$ ) and are at angles of $45^{\circ}$ to the vertical. Of course, this is not the true corner flow but merely an adequate approximation of it.

A modification of this model was proposed by Buck and $S u$ [1989] to explain the narrow volcanic zone near the surface. Although their results have important implications on the way the mantle upwelling focuses near the surface [Wilson, 1992], the area of partial melt in their model is not so different below depth $20 \mathrm{~km}$, making very minor differ- ences in our results. This is primarily because our surface wave data provide only gross features of the partial melt structure. We will consider the effects of such a model, specifically in the evaluation of the integral reported in Table 2 , and confirm that differences are very small.

One of the most important characteristics of equation (19) is that $\varepsilon_{f}$ is proportional to $v_{0}^{1 / n}$. Since $v_{0} \approx u_{F} / \pi$ according to a simple corner flow model, where $u_{F}$ is the full-spreading rate $\left(u_{F}=2 u\right)$, it follows that $\varepsilon_{f}$ is proportional to $u_{F}^{1 / n}$. This is in contrast to the thermal effect which shows a constant asymptotic value at large $u_{F}$. It is this difference that enable us to distinguish thermal from melting effects in the seismic data.

\section{Data Analysis}

The central data in this paper are shown in Figures 1a and 1b. Figure 1a shows the sampled location of data plotted in Figure 1b. Note, however, that data from slow spreading ridges with $u_{f} \leq 2 \mathrm{~cm} \mathrm{yr}^{-1}$ are excluded in the plot and are not used for analysis. Figure $\mathrm{lb}$ shows the $\log$-log plots of $\varepsilon$ as a function of spreading rate at four depths $(38,66,90$, and $110 \mathrm{~km}$ ) under ridge axes. Location (latitude and longitude), spreading rate, and $\varepsilon$ at four depths are given in Table 1 . In both Figures $1 \mathrm{a}$ and $\mathrm{lb}$, the data from the three major oceans are distinguished by different symbols, but they are not relevant to the following analysis.

\section{S velocity vs. Spreading Rate (Raw Data)}
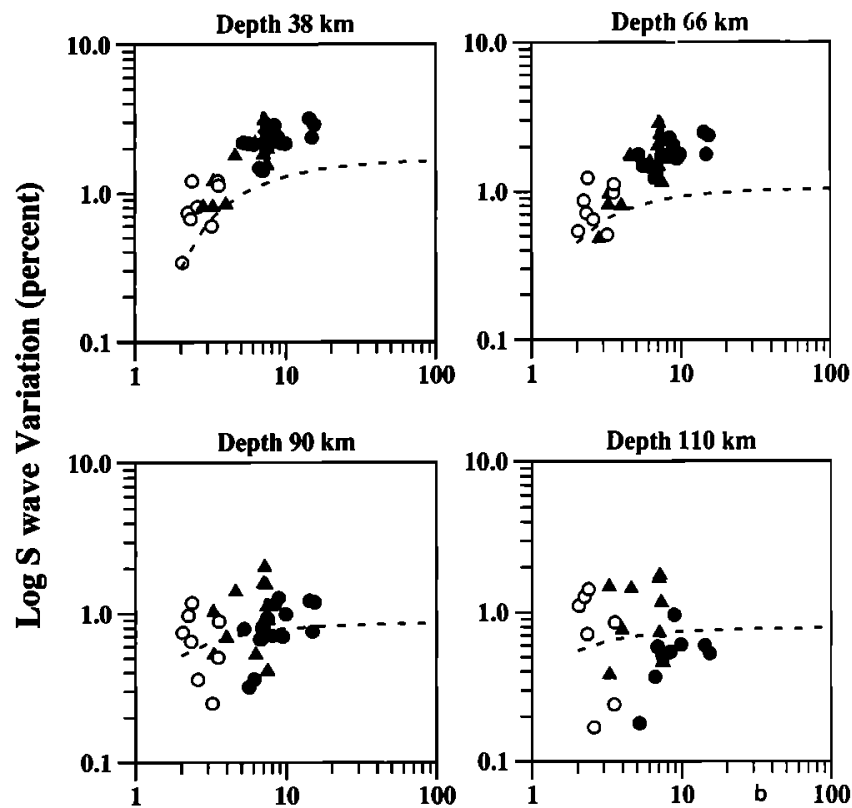

Full Spreading Rate $(\mathrm{cm} / \mathrm{y})$

Figure 1b. Log-log plot of data given in Table 1. There are 36 points after excluding data with spreading rate below $2 \mathrm{~cm}$ $\mathrm{yr}^{-1}$. The linear trend in the plots is clear at $38 \mathrm{~km}$ and $66 \mathrm{~km}$ but is very weak at $90 \mathrm{~km}$ and is probably nonexistent at 110 $\mathrm{km}$. Dashed lines are for the thermal model. The trend in data at shallow depths, especially at $66 \mathrm{~km}$, cannot be explained by thermal effects, and this discrepancy will be the basic information to estimate the amount of partial melts under the ridge axes. 
Table 1. Data of Sampled Ridge Locations Full-Spreading Rate, and $\varepsilon$ at Four Depths

\begin{tabular}{|c|c|c|c|c|c|c|c|}
\hline Location & Latitude & Longitude & $u_{F} \operatorname{cm~} \mathrm{yr}^{-1}$ & $38 \mathrm{~km}$ & $66 \mathrm{~km}$ & $90 \mathrm{~km}$ & $110 \mathrm{~km}$ \\
\hline SW Indian & -53.5 & 9.6 & 1.4 & 0.45 & 0.52 & 0.48 & 0.50 \\
\hline SW Indian & -52.3 & 14.9 & 1.4 & 0.05 & 0.24 & 0.52 & 0.87 \\
\hline SW Indian & -52.8 & 22.2 & 1.4 & 0.70 & 0.65 & 0.50 & 0.46 \\
\hline SW Indian & -29.4 & 60.8 & 1.4 & 1.44 & 1.15 & 0.46 & -0.03 \\
\hline SW Indian & -49.8 & 29.8 & 1.4 & 1.15 & 1.02 & 0.58 & 0.33 \\
\hline SW Indian & -46.1 & 34.8 & 1.4 & 0.87 & 0.82 & 0.61 & 0.62 \\
\hline SW Indian & -34.3 & 55.2 & 1.4 & 0.91 & 0.70 & 0.25 & -0.08 \\
\hline SW Indian & -43.4 & 39.7 & 1.4 & 0.85 & 0.80 & 0.68 & 0.82 \\
\hline SW Indian & -37.9 & 49.4 & 1.4 & 0.50 & 0.31 & 0.05 & -0.09 \\
\hline Mid-Atlantic & 60.2 & 330.2 & 2.0 & 0.34 & 0.54 & 0.75 & 1.11 \\
\hline Mid-Atlantic & 34.4 & 321.9 & 2.2 & 0.74 & 0.87 & 0.97 & 1.27 \\
\hline Mid-Atlantic & 50.1 & 330.1 & 2.3 & 0.07 & 0.03 & -0.13 & -0.27 \\
\hline Mid-Atlantic & 44.8 & 331.6 & 2.3 & 0.67 & 0.72 & 0.65 & 0.72 \\
\hline Mid-Atlantic & 29.8 & 317.5 & 2.4 & 1.21 & 1.24 & 1.18 & 1.43 \\
\hline Mid-Atlantic & 20.2 & 314.3 & 2.6 & 0.81 & 0.65 & 0.36 & 0.17 \\
\hline Central Indian & 5.1 & 61.0 & 2.8 & 0.81 & 0.48 & 0.08 & -0.17 \\
\hline Central Indian & 1.4 & 66.6 & 3.3 & 0.81 & 0.81 & 1.02 & 1.48 \\
\hline Mid-Atlantic & -49.8 & 353.1 & 3.2 & 0.60 & 0.51 & 0.25 & 0.07 \\
\hline Central Indian & 6.7 & 68.4 & 3.3 & 1.21 & 0.96 & 0.53 & 0.38 \\
\hline Mid-Atlantic & -17.0 & 346.0 & 3.5 & 1.21 & 0.98 & 0.51 & 0.24 \\
\hline Mid-Atlantic & -25.8 & 346.6 & 3.6 & 1.13 & 1.12 & 0.89 & 0.85 \\
\hline Central Indian & -15.6 & 67.1 & 4.0 & 0.84 & 0.80 & 0.69 & 0.76 \\
\hline Central Indian & -21.9 & 68.0 & 4.6 & 1.79 & 1.75 & 1.40 & 1.43 \\
\hline Pacific-Antarctica & -63.4 & 170.9 & 5.2 & 2.19 & 1.77 & 0.79 & 0.18 \\
\hline Pacific-Antarctica & -65.8 & 182.4 & 5.6 & 2.17 & 1.50 & 0.32 & -0.50 \\
\hline Pacific-Antarctica & -64.3 & 190.5 & 6.1 & 2.13 & 1.47 & 0.36 & -0.40 \\
\hline SE Indian & -29.4 & 74.9 & 6.2 & 2.16 & 1.56 & 0.53 & -0.13 \\
\hline Pacific-Antarctica & -62.6 & 199.7 & 6.7 & 1.47 & 1.23 & 0.67 & 0.37 \\
\hline Pacific-Antarctica & -62.1 & 204.1 & 6.9 & 1.42 & 1.27 & 0.80 & 0.59 \\
\hline SE Indian & -57.1 & 147.7 & 7.0 & 2.22 & 2.02 & 1.58 & 1.68 \\
\hline SE Indian & -41.8 & 85.4 & 7.1 & 1.81 & 1.48 & 0.90 & 0.73 \\
\hline SE Indian & -53.9 & 142.4 & 7.2 & 3.08 & 2.86 & 2.04 & 1.77 \\
\hline SE Indian & -49.4 & 135.2 & 7.3 & 2.73 & 2.42 & 1.54 & 1.16 \\
\hline SE Indian & -45.0 & 95.0 & 7.4 & 2.66 & 2.18 & 1.11 & 0.46 \\
\hline Pacific-Antarctica & -58.9 & 210.6 & 7.4 & 2.07 & 1.72 & 0.93 & 0.51 \\
\hline SE Indian & -49.2 & 124.7 & 7.5 & 1.54 & 1.15 & 0.41 & -0.08 \\
\hline SE Indian & -48.2 & 104.9 & 7.5 & 1.97 & 1.65 & 0.90 & 0.46 \\
\hline Pacific-Antarctica & -56.2 & 220.2 & 8.0 & 2.27 & 1.74 & 0.71 & 0.09 \\
\hline Pacific-Antarctica & -55.6 & 232.5 & 8.3 & 2.85 & 2.28 & 1.15 & 0.54 \\
\hline Pacific-Antarctica & -51.5 & 242.5 & 8.9 & 2.36 & 2.02 & 1.27 & 0.96 \\
\hline Pacific-Antarctica & -45.1 & 246.8 & 9.4 & 2.17 & 1.65 & 0.70 & 0.09 \\
\hline Cocos & 11.9 & 255.7 & 9.9 & 2.15 & 1.77 & 0.99 & 0.61 \\
\hline East Pacific Rise & -7.2 & 252.8 & 14.2 & 3.17 & 2.48 & 1.22 & 0.60 \\
\hline East Pacific Rise & -13.1 & 248.8 & 14.9 & 2.37 & 1.77 & 0.76 & 0.17 \\
\hline East Pacific Rise & -20.2 & 246.4 & 15.5 & 2.90 & 2.36 & 1.19 & 0.53 \\
\hline
\end{tabular}

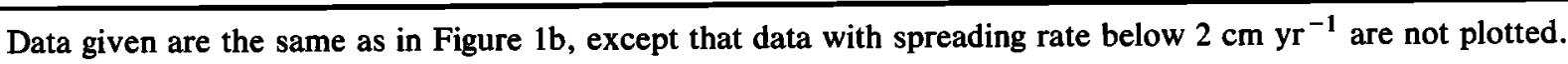

In the two shallow results ( 38 and $66 \mathrm{~km}$ ), there are clear linear trends. This trend clearly does not exist for results at $110 \mathrm{~km}$ and is weak at best at $90 \mathrm{~km}$. Along with data, theoretical curves for the thermal models are shown by dashed lines. They are obtained from (5) and (8). In this study, the parameters used for evaluation were $(\partial \ln \beta / \partial T)_{f}$ $=-0.007$ (percent $/ \mathrm{K})$ [Anderson, 1989], $(\partial \ln \beta / \partial f)=-2$ [Sato et al., 1989] and $\kappa=10^{-2}\left(\mathrm{~cm}^{2} \mathrm{~s}^{-1}\right)$. The dependence of seismic velocity on melting could conceivably be more extreme if the olivine-basalt system has sufficiently anisotropic surface energies leading to a very different microscopic texture than usually assumed [Waff and Faul, 1992]. We will return to this point in the discussion.

It should be kept in mind when interpreting Figure $1 \mathrm{~b}$ that since $T_{0}$ is not known, the curves could move in the vertical direction. The curves are shown to indicate that gradients in data cannot be matched by gradients in the thermal models. It is evident from the figure that, while results at both $38 \mathrm{~km}$ and $66 \mathrm{~km}$ support the evidence of partial melts, the strongest argument for a partial melting effect is found for the data at $66 \mathrm{~km}$.

The data obviously provide constraints on the depth to the solidus $z_{0}$. Unfortunately, the poor depth resolution of seismic data $( \pm 50 \mathrm{~km})$ limits greatly the precision, with which $z_{0}$ can be determined. Since there is a marked difference between 66 and $90 \mathrm{~km}$, a value of $60 \mathrm{~km}$ is plausible, but a value as high as $100 \mathrm{~km}$ cannot be excluded. This value is consistent with petrologically inferred value previously [e.g., Hess, 1992].

The most serious uncertainty arises from the existence of 
$T_{0}$ in the evaluation of thermal effects. One way to circumvent this problem is to estimate the term with $T_{0}$ from seismic data by averaging oceanic areas. An oceanic average, rather than the global average, may provide a better basis for evaluation of absolute temperature effects. Another approach is to recast the data into "relative" data and remove $T_{0}$ from equations altogether. In this paper, we adopt the latter approach.

\section{Relative Data}

First, we turn the data $\varepsilon$ into relative data by taking the difference between $\varepsilon$ and $\varepsilon_{\text {ref }}$ which we take to have the value of $3 \mathrm{~cm} \mathrm{yr}^{-1}$. Actually, we use the average value of data within $2 \leq u \leq 4 \mathrm{~cm} \mathrm{yr}^{-1}$ as the reference at $u=3 \mathrm{~cm}$ $\mathrm{yr}^{-1}$. The data to be examined will be

$$
\Delta \varepsilon=\varepsilon-\varepsilon_{\text {ref }} \equiv \Delta \varepsilon_{f}+\Delta \varepsilon_{T}
$$

where the subscript ref means the reference value. The new parameters $\Delta \varepsilon_{f}$ and $\Delta \varepsilon_{T}$ are partial melting and thermal parts of $\Delta \varepsilon$, respectively. We will evaluate $\Delta \varepsilon_{T}$ using the conductive cooling model which can now be done since it does not contain $T_{0}$.

Through the above process, we discard the slow spreading rate $\left(<2 \mathrm{~cm} \mathrm{yr}^{-1}\right)$ data which contain large scatter. The large scatter may arise from the episodicity of volcanism in space and time for slow spreading ridges. This is inferred from seafloor observations, particularly for parts of the midAtlantic ridge [Karson et al., 1987; Pockalny et al., 1988] and supported by observations of strong gravity and topography variation along the ridge axis and by theoretical modeling [Scott and Stevenson, 1989]. This is outside the realms of the simple model developed here. Moreover, surface wave dispersion data [Zhang and Tanimoto, 1991] report an unusually thick lithosphere for slow spreading ridges. A simple one-dimensional conduction model does not work in such a case. In any case, a simple model which we are using in this paper should be more relevant to faster-spreading rate ridges.

Theoretical formula for $\Delta \varepsilon_{T}$ is given by

$$
\begin{aligned}
\Delta \varepsilon_{T}=-\left(T_{m}-T_{s}\right) \int d v K\left(\frac{\partial \ln \beta}{\partial T}\right)_{f}\left\{\operatorname{erfc}\left(\frac{z}{2 \sqrt{2 \kappa x / u_{F, \text { ref }}}}\right)\right. \\
\left.-\operatorname{erfc}\left(\frac{z}{2 \sqrt{2 \kappa x / u_{F}}}\right)\right\}, \quad \text { (20) }
\end{aligned}
$$

which does not contain $T_{0}$ and is thus computable with some confidence. In our analysis, since we take the reference at 3 $\mathrm{cm} \mathrm{yr}^{-1} u_{F, \text { ref }}=3 \mathrm{~cm} \mathrm{yr}^{-1}$.

The formula for partial melting becomes

$$
\Delta \varepsilon_{f}=\left(\frac{\eta_{l}}{k_{0} \Delta \rho g}\right)^{1 / n} \Omega\left(z_{0}\right)\left(u^{1 / n}-u_{\mathrm{ref}}^{1 / n}\right)
$$

where

$$
\begin{aligned}
\Omega\left(z_{0}\right)=\int d v K\left(\frac{\partial \ln \beta}{\partial f}\right)_{T} & \left(1-\frac{z}{z_{0}}\right)^{1 / n} \\
& \cdot \Theta(z-|x|) \Theta\left(z_{0}-z\right) .
\end{aligned}
$$

The formula for $\Delta \varepsilon_{f}$ is in the form:

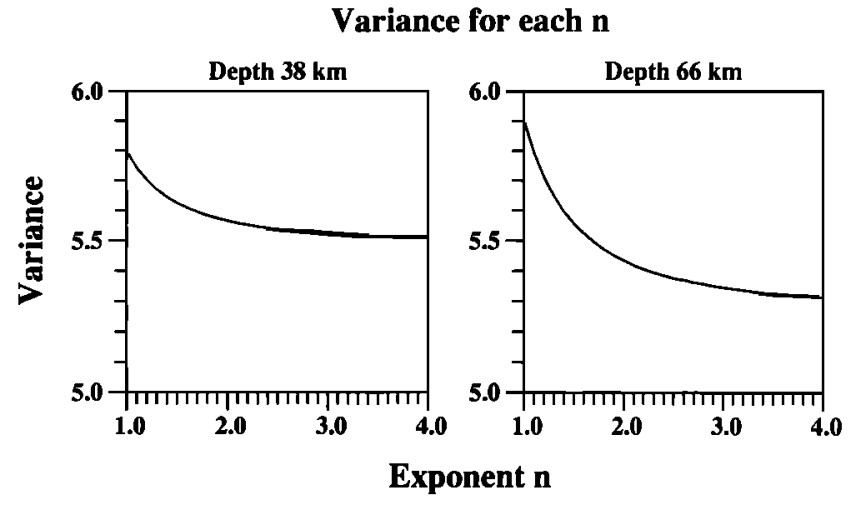

Figure 2. Variance $(J)$ versus the exponent $n$. The range below $n=2$ is not favored by the data. Although the range favored by theoretical models for permeability-porosity law $(2 \leq n \leq 3)$ is not at the minimum, it is within a broad region of low variance.

$$
\Delta \varepsilon_{f}=\gamma\left(u^{\delta}-u_{\mathrm{ref}}^{\delta}\right),
$$

where $\gamma$ is given below in (23) and $\delta=1 / n$ and both are to be determined by data. The data analysis proceeds in the following way; first, we form the relative data and subtract the thermal contribution to the relative data (equation (20)). We then have a set of data points which should be written as $\Delta \varepsilon_{f}^{(i)}$ for $i=1,2, \cdots, N$ where $N$ is the number of data. For each data point, we know the corresponding spreading rates $u_{i}$ (Table 1), which we obtained from DeMetz et al. [1990]. We then determine $\gamma$ and $\delta$ by minimizing

$$
J=\sum_{i=1}^{N}\left\{\Delta \varepsilon_{f}^{(i)}-\gamma\left(u_{i}^{\delta}-u_{\mathrm{ref}}^{\delta}\right)\right\}^{2},
$$

which requires the nonlinear parameter fit. This can be readily done by computing $\partial J / \partial \gamma$ and $\partial J / \partial \delta$ analytically, but since the problem has only two parameters, one can also understand global properties of $J(\gamma, \delta)$ fairly easily.

Figure 2 shows the plot of $J$ against $n$, which is the inverse of $\delta$. The minimum is found at higher $n$, although the small change of the variance with $n$ argues against attributing much significance to this. In fact, the straightforward nonlinear least squares fit converges to the minimum at $n$ larger than 10 , but the variance at $n=3$ is not so different from the global minimum value. There is some evidence against a low value $(n<2)$. This has some significance because a model with $n=1$ was suggested by Kohlstead [1992], based on experimental studies of partial melts, which is clearly not favored by our data. In the following, since $J$ has such a broad region of low values, we shall adopt the physically plausible $2 \leq n \leq 3$.

Figure 3 shows four curves that correspond to $n=1.5,2$, 2.5 , and 3 with the relative data $\Delta \varepsilon_{f}^{(i)}(i=1,2, \cdots)$. Clearly, it is hard to distinguish them. Estimates for $\gamma$, which is defined by

$$
\gamma \equiv\left[\frac{\eta_{l}}{k_{0} \Delta \rho g}\right]^{1 / n} \Omega\left(z_{0}\right),
$$

varies from $0.0037(n=2)$ to $0.0075(n=3)$ for the results at $38 \mathrm{~km}$ and from $0.0051(n=2)$ to $0.010(n=3)$ for the 


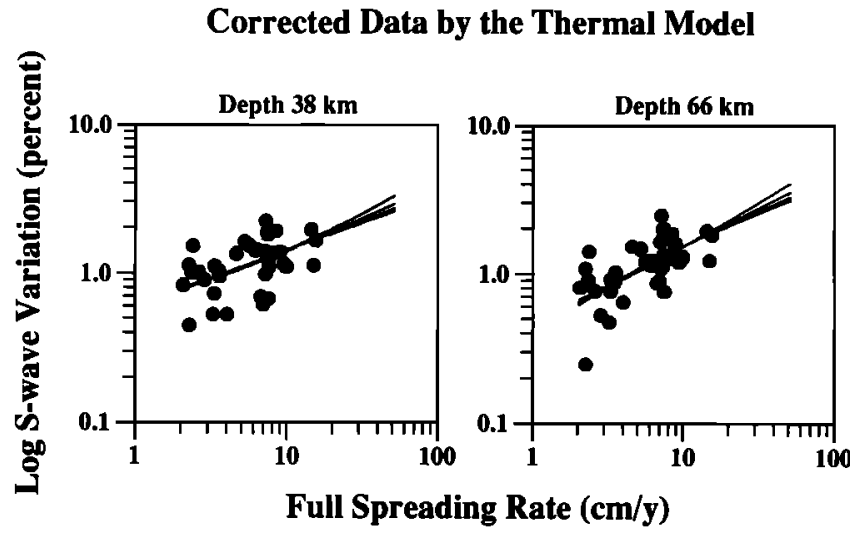

Figure 3. Relative data $\Delta \varepsilon_{f}^{(i)}$ versus four curves for $n=$ $1.5,2,2.5$, and 3 . Relative data were formed by referencing data to a value at $u_{F}=3 \mathrm{~cm} \mathrm{yr}^{-1}$ and subtracting thermal contributions (equation (20)). Each case with the minimum variance $(J)$ for $n=3,2.5,2$, and 1.5 is plotted from top to bottom. Although some recent experimental studies suggested $n=1$, there is a suggestion that $n \leq 2$ is not favored by our seismic data.

results at $66 \mathrm{~km}$. Since unit for $u_{F}$ was $\mathrm{cm} \mathrm{yr}^{-1}$, time unit for these numbers is also year. We use $\Delta \rho=0.5 \mathrm{~g} \mathrm{~cm}^{-3}, g=$ $980 \mathrm{~cm}^{2} \mathrm{~s}^{-1}$ and $\eta_{l}=100$ poise [Kushiro, 1986] for numerical evaluation of $k_{0}$. For $\Omega\left(z_{0}\right)$, we evaluate the integral in (22) numerically. In Table 2 we tabulate the results of this integration for six cases of $z_{0}$ (depth to the solidus $50-100 \mathrm{~km}$ ) at two target depths 38 and $66 \mathrm{~km}$ (differences from the kernel $K$ ). In the notation list, case $A$ corresponds to the corner flow model (14), and case B corresponds to the modified flow such as the one proposed by Buck and $S u$ [1989]. The major difference between the two models is in the top $20 \mathrm{~km}$ and has small effects on our results.

With those assumptions on parameters, we obtain the estimate for $k_{0}$ as

Table 2. Evaluation of Omega in (22)

\begin{tabular}{rrrrr}
\hline & \multicolumn{2}{c}{$38 \mathrm{~km}$} & \multicolumn{2}{c}{$66 \mathrm{~km}$} \\
\cline { 2 - 3 } \cline { 5 - 5 }$z_{0}$ & $n=2$ & $n=3$ & $n=2$ & $n=3$ \\
\hline \multicolumn{5}{c}{ Corner Flow Model } \\
50. & 0.396 & 0.485 & 0.136 & 0.179 \\
60. & 0.474 & 0.572 & 0.229 & 0.298 \\
70. & 0.524 & 0.621 & 0.342 & 0.442 \\
80. & 0.545 & 0.636 & 0.450 & 0.571 \\
90. & 0.541 & 0.617 & 0.534 & 0.664 \\
100. & 0.520 & 0.581 & 0.591 & 0.721 \\
& & & & \\
50. & 0.332 & 0.418 & 0.135 & 0.178 \\
60. & 0.419 & 0.515 & 0.227 & 0.297 \\
70. & 0.476 & 0.572 & 0.340 & 0.441 \\
80. & 0.503 & 0.592 & 0.449 & 0.570 \\
90. & 0.502 & 0.577 & 0.533 & 0.663 \\
100. & 0.485 & 0.546 & 0.590 & 0.720 \\
\hline
\end{tabular}

The parameter $z_{0}$ is the depth of solidus and $n$ is the exponent in the permeability-porosity relation. The main difference is whether the top $20 \mathrm{~km}$ of the Earth is included.

$$
k_{0}=10^{-8}-10^{-10} \mathrm{~m}^{2} .
$$

For a grain size of $1 \mathrm{~mm}, k_{0}$ was estimated to be $10^{-10} \mathrm{~m}^{2}$ by previous studies [Maaloe and Scheie, 1982; Scott and Stevenson, 1989]. Since the permeability is expected to be proportional to the square of the mean grain size [e.g., Turcotte and Ahern, 1978], this estimate of $k_{0}$ is perfectly consistent with $a=1-10 \mathrm{~mm}$.

\section{Model Implications}

With the estimated $k_{0}, n$, and $z_{0}$, we can calculate how porosity (partial melt distribution) and fluid velocity $\left(v_{l}\right)$ vary with depth and spreading rates. In Figures 4 and 5, we show the depth variation of porosity and fluid (magma) velocity based on our estimates in the previous section. Figure 4 shows the case for $z_{0}=80 \mathrm{~km}$, while Figure 5 shows the case for $z_{0}=100 \mathrm{~km}$. In each case, estimates for $n=2$ and for $n=3$ are shown.

Three solid lines in each figure correspond to the cases $u_{F}=5,10$, and $15 \mathrm{~cm} \mathrm{yr}^{-1}$, in all cases from left to right. Computed surface values vary between 1 and $2 \%$ for porosity and between 30 and $80 \mathrm{~cm} \mathrm{yr}^{-1}$ for fluid velocity. Depth dependence of porosity is $\left(1-z / z_{0}\right)^{1 / n}$ and that of fluid velocity is $\left(1-z / z_{0}\right)^{1-1 / n}$. Since $n=2$ or 3 , both parameters quickly reach asymptotic values above $z_{0}$ and do not change very much up to the surface. Because of this, depth to the solidus does not seem to make much difference in terms of the range of values for porosity and fluid velocity.

Spreading rate dependence is also similar; porosity is proportional to $u^{1 / n}$, and fluid velocity is proportional to $u^{1-1 / n}$. Because of this, a factor of difference in spreading rate by three in the figures $\left(5 \mathrm{~cm} \mathrm{yr}^{-1}\right.$ versus $\left.15 \mathrm{~cm} \mathrm{yr}^{-1}\right)$ affects both porosity and fluid velocity by about 2 , a relatively small change compared with the range of estimates for $k_{0}$. This is not surprising because porosity and fluid velocity can be written in terms of $\gamma$ and the range of estimate for $\gamma$ is much tighter than that for $k_{0}$. This is because removal of the exponent $1 / n$ from $\gamma$ is required to make an estimate for $k_{0}$.

It may be of some interest to know how quickly melt reaches the surface from $z_{0}$. In our model, analytical integration for this ascent time $\tau$ is possible, and it yields

$$
\tau=n z_{0} / v_{l}(0)
$$

where $v_{l}(0)$ is the value of $v_{l}$ at the surface. Explicitly, this is

$$
v_{l}(0)=\left(\frac{\eta_{l}}{k_{0} \Delta \rho g}\right)^{-1 / n} u^{1-1 / n} .
$$

If we take $v_{l}(0) \approx 50 \mathrm{~cm} \mathrm{yr}^{-1}$, we get

$$
\tau \approx 10^{5} \text { years. }
$$

There may be a factor of 5 variations, depending on various parameters, but $\tau$ seems to fall at about $10^{5}$ years.

Geochemical estimates [e.g., Gill and Condomines, 1992] for ascent times of magma are between a few decades and thousands of years. Times for ascent and for differentiation (meaning magma chamber processes) can be separately estimated and tend to be comparable. There is some difficulty in knowing how to compare this with our estimate 


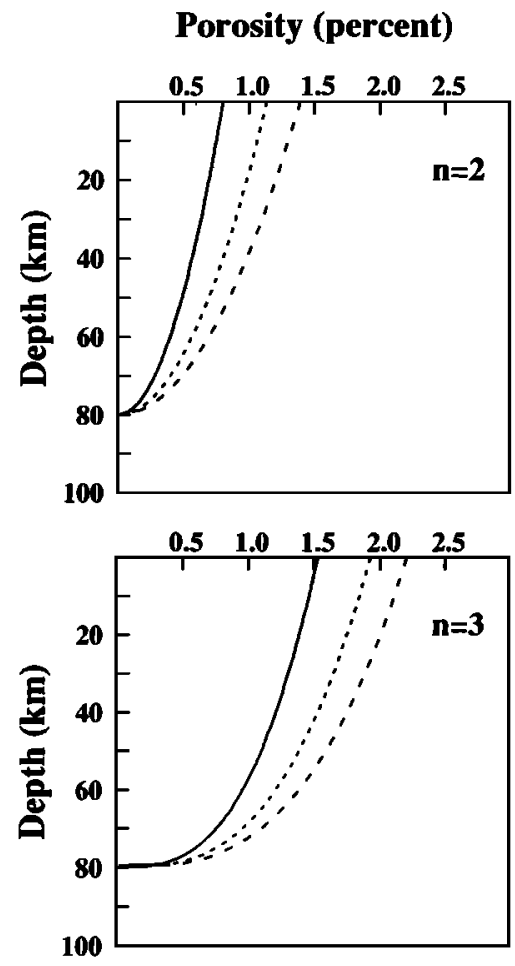

Fluid Velocity $(\mathrm{cm} / \mathrm{y})$
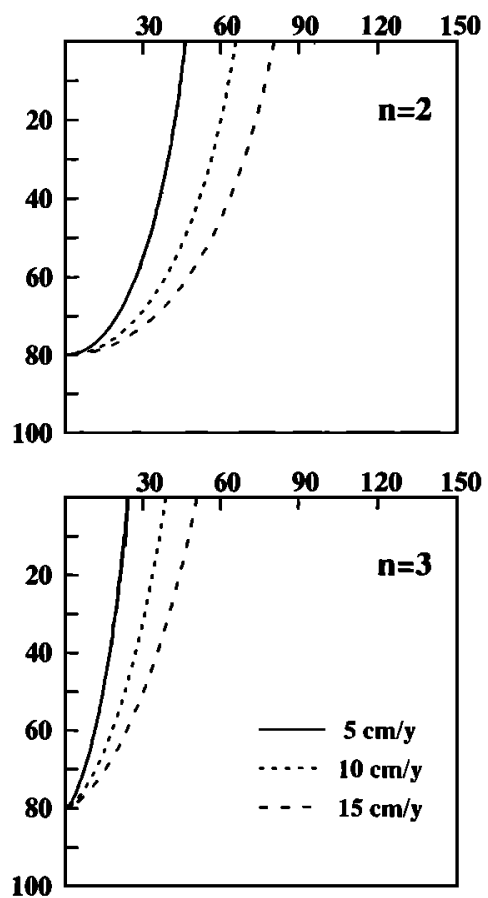

$\mathrm{Z0}=80 \mathrm{~km}$

Figure 4. Porosity and fluid velocity models computed for $z_{0}=80 \mathrm{~km}$. The top plots show $n=2$ and the bottom plots show $n=3$. Three curves are for different cases of (full) spreading rates.

without developing a detailed integrated geochemicalgeophysical model to evaluate exactly what isotopic observations are expected for a specified sequence of physical processes. The difficulty is further compounded by the possibility that some of the melt is delivered promptly along macroscopic channels, while other melt may be forced along slow permeability pathways. Chemical disequilibrium is another way of addressing this possibility [Spiegelman and Kenyon, 1992]. The biggest problem lies in our lack of knowledge concerning how melt migration makes the transition from microscopic permeability to macroscopic transport along channels.

Additional complication lies in the possibility that the microscopic melt distribution is not the usually assumed interconnecting tubules but includes some partial facewetting [Waff and Faul, 1992]. This would have the effect of producing a larger seismic velocity reduction for a given (small) melt fraction. Our melt fraction estimates would then be too high. Since there is no doubt about how much melt migrates, this would mean a much higher permeability than we obtain. This is difficult to envisage unless one supposes that some of the permeability is in the form of macroscopic channels, even at great depth. Further work is needed to assess this.

\section{Discussion}

Since our approach is a global approach, regional variations of the melting regimes beneath individual ridges are not explored. The only parameter considered is the spreading rate. It is now well known, however, that bathymetric, tomographic, and geochemical evidence suggests that sub- solidus temperature varies from region to region independent of spreading rate [e.g., Forsyth, 1992]. Thus a question may arise as to what the effects of variations in subsolidus temperature will have in regard to the modeling. The answer to this is fairly straightforward; what has been demonstrated clearly, especially in Figure $1 b$, is the predominance of partial melting effects on shallow $S$ wave velocity structure. Systematic trend in the 38- and 66-km maps is most naturally explained by existence of partial melts. The effects of subsolidus temperature variations are mostly likely the cause of data scatter in Figure 1b. Of course, one can ask further whether a fine structure due to subsolidus temperature variation can be seen in deeper maps. There seem to be some suggestions, but scatter in the data is too large to reach a convincing conclusion from the current velocity model. We need a better seismic model to examine such questions.

There have been some questions raised against the model by Zhang and Tanimoto [1992, 1993] because it does not satisfy travel times of some body wave phases, specifically SS-S data [Su et al., 1992]. It is argued that heterogeneity is confined to shallow depths because of overdamping in the process of inversion, sometimes called the squeezing effect. This argument has some merits in it, because heterogeneity in the transition zone in the model of Zhang and Tanimoto is probably not right. It lacks the long wavelength $(l=2)$ pattern in the transition zone that has been recovered consistently in other studies [e.g., Masters et al., 1982; Woodhouse and Dziewonski, 1984; Nataf et al., 1986; Tanimoto, 1988, 1990]. However, such "squeezing" of heterogeneity cannot occur below $200-300 \mathrm{~km}$. This is because short period Rayleigh waves used in Zhang and Tanimoto 


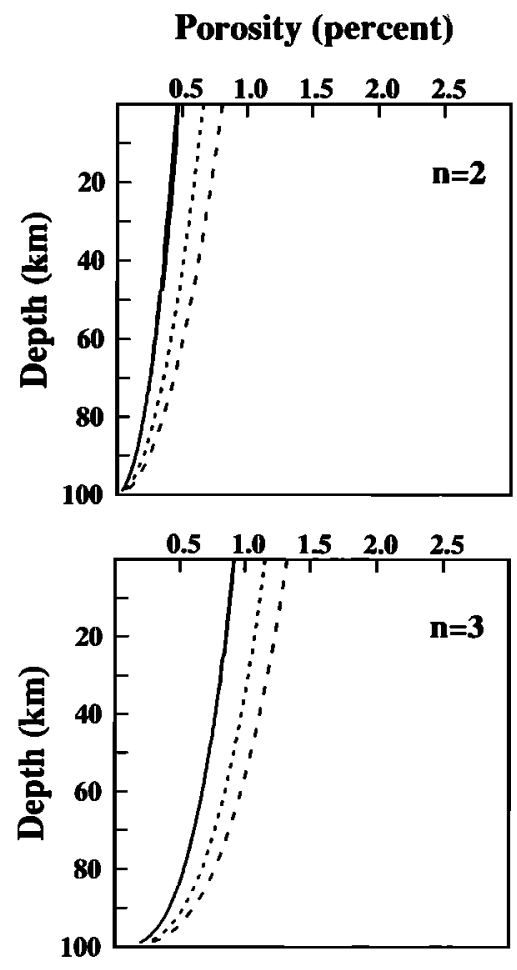

Fluid Velocity $(\mathrm{cm} / \mathbf{y})$
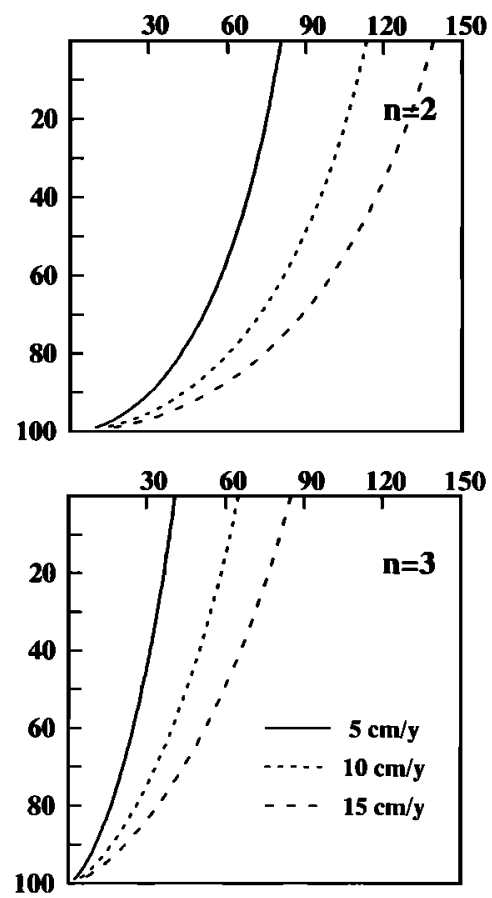

100

\section{$\mathrm{Z0}=100 \mathrm{~km}$}

Figure 5. Same as Figure 4, except for $z_{0}=100 \mathrm{~km}$.

have larger sensitivity to depth between 100 and $200 \mathrm{~km}$ than shallower depth. If a significant "squeezing" occurs, Rayleigh wave data will be violated too much. Thus Rayleigh waves will stop such squeezing effects below about 200-300 $\mathrm{km}$. Also, examination of surface wave models by SS-S data is not necessarily straightforward, since both phases have turning points at considerably deeper depths than $300 \mathrm{~km}$, possibly in the lower mantle. Thus the well-known, large heterogeneity in the transition zone may have some effects on them. It is clearly more desirable that a high-resolution model, which incorporates both data sets, is used, but such a model does not yet exist.

More importantly, however, the seismic model by Zhang and Tanimoto is not so different from other models as far as the depth range $(0-100 \mathrm{~km})$ is concerned. The pattern in the long-wavelength range, that is, the spherical harmonic angular degree that is less than 12 , is similar and amplitude difference is about $20-30 \%$ for the depth range. Since our analysis is based on $\log -\log$ plots, those differences have relatively small effects.

\section{Conclusions}

From the variations of seismic velocity under ridge axes as a function of spreading rate, we demonstrated that existence of partial melt was required. On the basis of a simple model of partial melting (porous flow by Darcy's law), we reach the following conclusions:

1. Depth to the solidus is about $60-100 \mathrm{~km}$, which is not surprising, based on previous petrological evidences but is nonetheless important to confirm directly by seismic data.

2. If the exponent in the permeability-porosity relation $k(f)=k_{0} f^{n}$ is $2 \leq n \leq 3$, the range for $k_{0}$ is $k_{0}=$
$10^{-10}-10^{-8} \mathrm{~m}^{2}$. Estimates for $k_{0}$ is slightly larger than previous estimates, but a grain size of $1-10 \mathrm{~mm}$ rather than $1 \mathrm{~mm}$ can explain our results.

These two conclusions are fairly direct conclusions from seismic observation. The following two statements can be made by applying the determined parameters to the model:

1. Estimated porosity and fluid velocity are 1-2\% and about $50 \mathrm{~cm} \mathrm{yr}^{-1}$, respectively. Depth and spreading rate dependence of porosity and fluid velocity were obtained analytically.

2. Ascent time of melt from solidus to the surface is of the order of $\mathrm{O}\left(10^{5}\right)$ years. This is somewhat larger than geochemical estimates, but its resolution requires development of more detailed geochemical-geophysical models, and thus it is beyond the scope of this paper.

\section{Notation}

$K$ resolution (averaging) kernel derived by seismic analysis.

$T$ temperature.

$T_{0}$ the global average temperature at each depth.

$T_{m}$ mantle temperature.

$T_{s}$ surface temperature.

$f$ porosity (filled with melt).

$g$ gravitational acceleration.

$k$ permeability.

$k_{0}$ constant in the permeability-porosity relation.

$m$ the degree of melting.

$m_{0}$ proportional constant in the formula for $m$.

$n$ exponent in the permeability-porosity relation.

$u$ half-spreading rate.

$u_{F}$ full-spreading rate. 
$v$ volume in the Earth.

$v_{0}$ mantle flow velocity below the solidus.

$v_{l}$ liquid (magma) velocity in the porous medium.

$v_{s}$ solid velocity in the porous medium.

$z_{0}$ depth to the solidus.

$\beta \quad S$ wave velocity.

$\delta \beta \quad S$ wave velocity perturbation.

$\beta_{g} \quad$ the global average of $S$ wave velocity at each depth.

$\varepsilon$ fractional perturbation of $S$ wave velocity from the global average.

$\varepsilon_{T} \quad$ portion of $\varepsilon$ due to temperature effects.

$\varepsilon_{f}$ portion of $\varepsilon$ due to partial melt.

$\eta_{l}$ viscosity of magma.

$\kappa$ thermal diffusivity.

$\rho_{l}$ density of magma.

$\rho_{s}$ density of solid.

$\tau$ time for melt to reach surface.

$\Delta \rho$ difference between $\rho_{s}$ and $\rho_{l}$.

$\Theta$ the Heaviside step function.

Acknowledgments. This work was supported by NSF grants OCE9296207 and EAR-9296218 (TT) and EAR-9017893 (DJS). Institute of Crustal Studies Contribution number 143-24CS at UCSB Division of Geological and Planetary Sciences contribution number 5349 at Caltech. The paper benefited greatly by the constructive reviews provided by Anne Sheehan, Charles Langmuir, an anonymous reviewer, and an associate editor.

\section{References}

Anderson, D. L., Theory of the Earth, Blackwell, Oxford, 1989.

Buck, W. R., and W. Su, Focused mantle upwelling below midocean ridges due to feedback between viscosity and melting, Geophys. Res. Lett., 16, 641-644, 1989.

DeMetz, C., R. G. Gordon, D. F. Argus, and S. Stein, Current plate motions, Geophys. J. Int., 101, 425-478, 1990.

Forsyth, D. W., Geophysical constraints on mantle flow and melt generation beneath mid-ocean ridges, in Mantle Flow and Melt Generation at Mid-Ocean Ridges, edited by J. P. Morgan et al., Geophys. Monogr. Ser., vol. 71, pp. 1-65, AGU, Washington, D. C., 1993.

Gill, J., and M. Condomines, Short-lived radioactivity and magma genesis, Science, 257, 1368-1376, 1992.

Hayes, D. E., Age-depth relationships and depth anomalies in the southwest Indian Ocean and South Atlantic Ocean, J. Geophys. Res., 93, 2937-2954, 1988.

Hess, P. C., Phase equilibria constraints on the origin of ocean floor basalts, in Mantle Flow and Melt Generation at Mid-Ocean Ridges, edited by J. P. Morgan, et al., Geophys. Monogr. Ser., vol. 71, pp. 67-102, AGU, Washington, D. C., 1993.

Karson, J. A., et al., Along axis variations in seafloor spreading in the MARK area, Nature, 328, 681-685, 1987.

Klein, E. M., and C. H. Langmuir, Global correlations of ocean ridge basalt chemistry and axial depth and crustal thickness, $J$. Geophys. Res., 92, 8089-8115, 1987.

Kohlstedt, D. L., Structure, rheology and permeability of partially molten rocks at low melt fractions, in Mantle Flow and Melt Generation at Mid-Ocean Ridges, edited by J. P. Morgan et al., Geophys. Monogr. Ser., vol. 11, pp. 103-121, AGU, Washington, D. C., 1993.

Kushiro, L., Viscosity of partial melts in the upper mantle, $J$. Geophys. Res., 91, 9343-9350, 1986.

Maaloe, S., and A. Scheie, The permeability-controlled accumulation of primary magma, Contrib. Mineral. Petrol., 81, 350-357, 1982.

Masters, T. G., T. H. Jordan, P. G. Silver, and F. Gilbert, Aspherical Earth structure from fundamental spheroidal mode data, Nature, 298, 609-613, 1982.

Mavko, G. M., Velocity and attenuation in partially molten rocks, $J$. Geophys. Res., 85, 5173-5189, 1980.

Nataf, H.-C., I. Nakanishi, and D. L. Anderson, Measurements of mantle wave velocities and inversion for lateral heterogeneity and anisotropy, 3, Inversion, J. Geophys. Res., 91, 7261-7307, 1986.

Parsons, B., and J. G. Sclater, An analysis of the variation of ocean floor bathymetry and heat flow with age, J. Geophys. Res., 82, 803-827, 1977.

Pockalny, R. A., R. S. Detrick, and P. J. Fox, Morphology and tectonics of the Kane transform from Sea Beam bathymetry data, J. Geophys. Res., 93, 3179-3193, 1988.

Sato, H., I. S. Sacks, and T. Murase, The use of laboratory velocity data for estimating temperature and partial melt fraction in the low velocity zone: Comparison with heat flow and electrical conductivity measurements, J. Geophys. Res., 94, 5689-5704, 1989.

Scott, D. R., and D. J. Stevenson, A self-consistent model of melting, magma migration and buoyancy-driven circulation beneath mid-ocean ridges, J. Geophys. Res., 94, 2973-2988, 1989.

Shankland, T. J., R. J. O'Connell, and H. S. Waff, Geophysical constraints on partial melts in the upper mantle, Rev. Geophys., $19,394-406,1981$.

Sheehan, A. F., and S. C. Solomon, Joint inversion of shear wave travel time residuals and geoid and depth anomalies for longwavelength variations in upper mantle temperature and composition along the mid-Atlantic ridge, J. Geophys. Res., 96, 19,981$20,009,1991$.

Spiegelman, M., and P. Kenyon, The requirements for chemical disequilibrium during magma migration, Earth Planet. Sci. Lett., 109, 611-620, 1992.

Spiegelman, M., and D. McKenzie, Simple 2-D models for melt extraction at mid-ocean ridges and island arcs, Earth Planet. Sci. Lett., 83, 137-152, 1987.

Su, W.-J., R. L. Woodward, and A. M. Dziewonski, Deep origin of mid-oceanic ridge velocity anomalies, Nature, 360, 149-152, 1992.

Tanimoto, T., The 3-D shear-wave structure in the mantle by overtone waveform inversion, II, Inversion of $X$ waves, $R$ waves, and $G$ waves, Geophys. J. R. Astron. Soc., 93, 321-334, 1988.

Tanimoto, T., Long wavelength $S$ wave velocity structure throughout the mantle, Geophys. J. R. Astron. Soc., 100, 327-336, 1990.

Toramaru, A., and N. Fujii, Connectivity of the melt phase in partially molten peridotite, J. Geophys. Res., 91, 9239-9252, 1986.

Turcotte, D. L., and J. L. Ahern, A porous flow model for magma migration in the asthenosphere, J. Geophys. Res., 83, 767-772, 1978.

Waff, H. S., and U. H. Faul, Effects of crystalline anisotropy on fluid distribution in ultramafic partial melts, J. Geophys. Res., 97, 9003-9014, 1992.

Wilson, D. S., Focused mantle upwelling beneath mid-ocean ridges: Evidence from seamount formation and isostatic compensation of topography, Earth Planet. Sci. Lett., 113, 41-55, 1992.

Wood, B. J., and D. A. Yuen, The role of lithosphere phase transitions on seafloor flattening at old ages, Earth Planet. Sci. Lett., 66, 303-314, 1983.

Woodhouse, J. H., and A. M. Dziewonski, Mapping the upper mantle: Three-dimensional modeling of earth structure by inversion of seismic waveforms, J. Geophys. Res., 89, 5953-5986, 1984.

Woodward, R. L., and G. Masters, Global upper mantle structure from long-period differential travel times, J. Geophys. Res., 96, 6351-6377, 1991

Zhang, Y.-S., and T. Tanimoto, Global Love wave phase velocity variation and its significance to plate tectonics, Phys. Earth Planet. Int., 66, 160-202, 1991.

Zhang, Y.-S., and T. Tanimoto, Ridges, hotspots and their interaction as observed in seismic velocity maps, Nature, 355, 45-49, 1992.

Zhang, Y.-S., and T. Tanimoto, High-resolution global upper mantle structure: Plate tectonics, J. Geophys. Res., 98, 9793-9823, 1993.

D. J. Stevenson, Division of Geological and Planetary Sciences, California Institute of Technology, Pasadena, CA 91125.

T. Tanimoto, Institute of Crustal Studies and Department of Geological Sciences, University of California, Santa Barbara, CA 93106.

(Received March 17, 1993; revised November 22, 1993; accepted November $30,1993$. 